\title{
Hepáticas (Marchantiophyta) de um fragmento de Mata Atlântica na Serra da Jibóia, Município de Santa Teresinha, BA, Brasil ${ }^{1}$
}

\author{
Emilia de Brito Valente ${ }^{2,4}$ e Kátia Cavalcanti Pôrto ${ }^{3}$
}

Recebido em 12/04/2005. Aceito em 27/09/2005

\begin{abstract}
RESUMO - (Hepáticas (Marchantiophyta) de um fragmento de Mata Atlântica na Serra da Jibóia, Município de Santa Teresinha, BA, Brasil). Este trabalho apresenta os resultados do levantamento das hepáticas de um fragmento de Mata Atlântica, no Município de Santa Teresinha, Bahia. Foram registradas 70 espécies pertencentes a 41 gêneros e 14 famílias: Aneuraceae (2), Bryopteridaceae (2), Calypogeiaceae (1), Cephaloziaceae (2), Geocalycaceae (2), Herbertaceae (1), Jubulaceae (4), Lejeuneaceae (37), Lepidoziaceae (4), Metzgeriaceae (2), Pallaviciniaceae (2), Plagiochilaceae (8), Radulaceae (2), Trichocoleaceae (1). A família Lejeuneaceae é representada por 53\% das espécies. A comunidade corticícola apresentou a maior riqueza específica (67\%), seguida pelas epífila (33\%) e epíxila (14\%). Cinco tipos de formas de crescimento foram reconhecidas: trama $(69 \%)$, talosa $(9 \%)$, tapete $(19 \%)$, pendente $(3 \%)$ e tufo $(1 \%)$. Os táxons registrados para a Serra da Jibóia correspondem àqueles mais característicos de florestas tropicais baixo montana e submontana.
\end{abstract}

Palavras-chave: briófitas, brioflora, hepáticas, Lejeuneaceae, distribuição geográfica

\begin{abstract}
Hepatics (Marchantiophyta) from a fragment of Atlantic Forest in Serra da Jibóia, in the Municipality of Santa Teresinha, Bahia State, Brazil). This paper provid the results of the survey of hepatics at Serra da Jibóia, remaining Atlantic Forest, Santa Teresinha Municipality, Bahia. Seventy especies were recorded belonging to 41 genera and 14 families: Aneuraceae (2), Bryopteridaceae (2), Calypogeiaceae (1), Cephaloziaceae (2), Geocalycaceae (2), Herbertaceae (1), Jubulaceae (4), Lejeuneaceae (37), Lepidoziaceae (4), Metzgeriaceae (2), Pallaviciniaceae (2), Plagiochilaceae (8), Radulaceae (2), Trichocoleaceae (1). The family Lejeuneaceae were represents for $52,9 \%$ of the species. Three substrates were colonized: living trunks (67\%), trunks in decomposition (14\%) and leaves (33\%). Five growth-forms were found: mat (69\%), thallose (9\%), carpet (19\%), pendent $(3 \%)$ and turf (1\%). The results are similar to these found in tropical rainforest lower montane and submontane.
\end{abstract}

Key words: bryophytes, bryoflora, liverworts, Lejeuneaceae, geographical distribution

\section{Introdução}

As briófitas são um importante componente das florestas tropicais úmidas, ambiente onde ocorre maior riqueza e exuberância deste grupo de plantas (Richards 1984). É estimado que na região neotropical ocorram 1350 espécies de hepáticas (Gradstein et al. 2001), sendo que, para o Brasil são reconhecidas ca. 600 espécies deste grupo (Gradstein \& Costa 2003). A região Atlântica costeira se revela como a mais rica do Brasil e a terceira do Neotrópico em diversidade de espécies, perdendo apenas para o norte dos Andes e América Central (Gradstein et al. 2001).

Gradstein \& Costa (2003) citam 120 espécies de hepáticas para a Bahia. Este número aumenta para um total 187 quando se adicionam os novos registros publicados recentemente por Bastos \& Yano (2002; 2003) e C.J.P. Bastos (dados não publicados).

Diversos trabalhos com enfoque taxonômico sobre este grupo de plantas vêm sendo desenvolvidos na Bahia, como Bastos \& Vilas Bôas-Bastos (2000a; b; c) e Bastos \& Yano (2003); que publicaram novas adições à flora de hepáticas; Bastos \& Yano (2002); que registraram um novo táxon para a ciência; e, mais recentemente, C.J.P. Bastos (dados não publicados), que descreve e ilustra 120 espécies da família Lejeuneaceae.

As hepáticas foram objeto de estudo em diferentes ecossistemas do Estado, como cerrado (Vilas BôasBastos \& Bastos 1998), caatinga (Bastos et al. 1998a),

\footnotetext{
1 Parte da Dissertação de Mestrado da primeira Autora

2 Programa de Pós-Graduação em Biologia Vegetal da Universidade Federal de Pernambuco, Av. Nelson Chaves s/n, CEP 50760-420, Recife, PE, Brasil

3 Universidade Federal de Pernambuco, Centro de Ciências Biológicas, Departamento de Botânica

4 Autor para correspondência: ebvalente@bol.com.br
} 
restinga (C.J.P. Bastos, dados não publicados) e campo rupestre (Harley 1995; Bastos et al. 1998b; 2000). Entretanto, verifica-se a ausência de trabalhos florísticos no ecossistema Mata Atlântica.

O presente trabalho apresenta a composição florística das hepáticas (Marchantiophyta) de um fragmento de Mata Atlântica situado na Serra da Jibóia, Município de Santa Teresinha, Bahia, Brasil, constituindo-se, portanto, em uma contribuição pioneira para o Estado.

\section{Material e métodos}

Área de estudo - A Serra da Jibóia, localiza-se no município de Santa Teresinha (BA) a uma latitude de $12^{\circ} 51^{\prime} \mathrm{S}$ e longitude de $39^{\circ} 28^{\prime} \mathrm{O}$, com 6 km de extensão. Apresenta como tipos vegetacionais trechos de caatinga na base, mata higrófila na encosta e, no topo, um afloramento rochoso de origem gnáissico-granítica. A mata higrófila desenvolve-se nas encostas entre 400 e $800 \mathrm{~m}$ de altitude, caracterizando-se como Floresta Ombrófila Densa Montana de acordo com Veloso \& Góes Filho (1982).

A precipitação pluviométrica na área possivelmente é superior à referida para o município de Santa Teresinha (568 $\mathrm{mm}$ anuais), devendo atingir pelo menos $1.100 \mathrm{~mm}$ anuais, que é o mínimo registrado para a mata higrófila sul-baiana (Gouvêa et al. 1976). O fato de estar inserida no semi-árido baiano faz da mata higrófila da Serra da Jibóia, um local singular, já que está isolada de outros fragmentos florestais do litoral pela caatinga que a circunda (Queiroz et al. 1996).

Alguns estudos já foram desenvolvidos na área, como o realizado por Queiroz et al. (1996) com a flora vascular ocorrente no afloramento rochoso do Morro da Pioneira, onde foram registradas 83 espécies, sendo as famílias mais representativas: Compositae, Orchidaceae, Leguminosae e Melastomataceae. No campo da entomologia, foram encontradas 14 espécies de Díptera inéditas para a ciência (Bravo 2001a; 2001b; $2002 ; 2003 ; 2004)$. Na área de micologia, foi realizado recentemente um levantamento de Aphyllophorales, no qual foram registradas 26 espécies (Góes Neto et al. 2003).

Amostragem e estudo do material - O material analisado foi proveniente de oito expedições realizadas pela autora entre 2001 e 2004 e de coleções depositadas no HUEFS (Herbário da Universidade Estadual de Feira de Santana, Feira de Santana, BA) e no ALCB (Herbário Alexandre Leal Costa, Instituto de Biologia-UFBA).
O material coletado nas expedições encontra-se depositado nos herbários HUEFS e UFP (Universidade Federal de Pernambuco, Recife, PE).

As amostras foram coletadas na margem e, preferencialmente, no interior da mata em locais de umidade mais elevada e com menor incidência de luz, ao longo de trilhas já existentes ou abertas, quando necessário. Foram observados os seguintes tipos de substratos, considerados preferenciais para briófitas: troncos em decomposição, troncos vivos (de 0-2 m de altura), folhas, rochas e solo. O método de coleta e herborização foi o tradicionalmente utilizado, descrito por Yano (1989). A expressão "espécie exclusiva” foi adotada para especificar aquela que só ocorreu em um único substrato na área de estudo.

A literatura utilizada para a identificação das espécies foi a seguinte: Bischler $(1962 ; 1964 ; 1967)$, Fulford (1963; 1966; 1968), Hell (1969), Reyes (1982), Lemos-Michel (1983; 2001), Onraedt (1985; 1988), Gradstein (1989), Teeuwen (1989), Reiner-Drehwald (1994; 1998; 2000), Costa \& Moura (1996), Grolle \& Reiner-Drehwald (1997), Heinrichs et al. (1998; 2000), Oliveira e Silva \& Yano (2000), Gradstein et al. (2001), Lemos-Michel \& Yano (1998), Bernecker-Lücking (1999), Gradstein \& Costa (2003), Bastos \& Vilas BôasBastos (2000a; b), Ilkiu-Borges \& Lisboa (2002a; b).

Duplicatas de amostras de difícil identificação foram enviadas para confirmação aos seguintes especialistas: Dr. Cid José Passos Bastos, da Universidade Federal da Bahia, Dra. Denise Pinheiro da Costa, do Instituto de Pesquisas Jardim Botânico do Rio de Janeiro e Dr. Jochen Heirinchs, da Universidade de Göttingen, Alemanha.

A distribuição geográfica e variação altitudinal das espécies foi baseada em Gradstein \& Costa (2003) e, quando necessário, em literatura específica. A identificação das formas de crescimento foi feita com base em Mägdefrau (1982) e Richards (1984). O sistema de classificação adotado foi o de CrandallStotler \& Stotler (2000).

Foi considerada como espécie de distribuição rara, aquela conhecida em 1-4 estados brasileiros, de distribuição moderada, a que ocorre em 5-9 estados e de distribuição ampla a que ocorre em 10-14 estados.

\section{Resultados e discussão}

O levantamento das hepáticas da Serra da Jibóia resultou em 70 espécies pertencentes a 41 gêneros e 14 famílias, sendo 21 referidas pela primeira vez para a Bahia (Tab. 1). Dentre as famílias de hepáticas 
citadas por Richards (1984) e Gradstein \& Pócs (1989) como as principais em levantamentos florísticos nas florestas tropicais, Jubulaceae, Lejeuneaceae, Lepidoziaceae, Plagiochilaceae e Radulaceae ocorreram na área de estudo, sendo bem representadas em número de espécies e/ou de ocorrência. As demais famílias, Aneuraceae, Bryopteridaceae, Calypogeiaceae, Cephaloziaceae, Herbertaceae, Metzgeriaceae e
Pallaviciniaceae, também presentes na área, embora com menor representatividade, são comuns nas florestas tropicais úmidas (Gradstein et al. 2001).

A família Lejeuneaceae foi a mais representativa com $53 \%$ do total das espécies, dentre as quais, seis são referidas pela primeira vez para o Estado (Tab. 1). Gradstein et al. (2001) relatam a elevada riqueza específica de Lejeuneaceae em florestas tropicais baixo

Tabela 1. Lista das espécies de hepáticas da Serra da Jibóia, Município de Santa Teresinha, Bahia, Brasil, com seus respectivos dados em relação ao substrato, forma de crescimento, variação altitudinal no Brasil e distribuição geográfica mundial e no Brasil. Substrato: $\mathrm{TV}=$ tronco vivo, $\mathrm{F}=$ folha, $\mathrm{TD}=$ tronco em decomposição; Forma de crescimento: $\mathrm{TR}=$ trama, $\mathrm{TP}=$ tapete, $\mathrm{TF}=$ tufo, $\mathrm{TL}=$ talosa, $\mathrm{P}=$ pendente; $*$ = novas referências para a Bahia; $-=$ não informado em exsicata.

\begin{tabular}{cccc}
\hline Famílias/Espécies & Substrato & Forma de \\
crescimento & $\begin{array}{c}\text { Variação altitudinal } \\
\text { no Brasil }\end{array}$ & $\begin{array}{c}\text { Distribuição mundial } \\
\text { e no Brasil }\end{array}$
\end{tabular}

ANEURACEAE
*Riccardia amazonica (Spruce) S.W. Arnell
*Riccardia fucoidea (Sw.) Schiffin.
BRYOPTERIDACEAE
Bryopteris filicina (Sw.) Nees
Bryopteris diffusa (Sw.) Nees
CALYPOGEIACEAE
*Calypogeia peruviana Nees \& Mont.
CEPHALOZIACEAE
*Cephalozia crassifolia (Lindenb. \& Gottsche)
Fulford
Odontoschisma falcifolium Steph.
GEOCALYCACEAE
*Leptoscyphus gibbosus (Tayl.) Mitt.
Lophocholea martiana Nees

\section{HERBERTACEAE}

Herbertus juniperoideus (Sw.) Grolle JUBULACEAE

Frullania apiculata (Reinw. et al.) Nees

Frullania beyrichiana (Lehm. \& Lindenb.) Lehm. \& Lindenb.

Frullania brasiliensis Raddi.

Frullania caulisequa (Nees) Nees

\section{LEJEUNEACEAE}

Anoplolejeunea conferta (Meissn. ex Spreng.) A. Evans

Ceratolejeunea cornuta (Lindenb.) Steph.

\begin{tabular}{ccc} 
TD & TL & $0-2200 \mathrm{~m}$ \\
TV/TD & TL & $0-1500 \mathrm{~m}$ \\
TV & P & $0-2000 \mathrm{~m}$ \\
TV/F & P & $0-1500 \mathrm{~m}$ \\
& & \\
TD & TR & $0-400 \mathrm{~m}$ \\
& & \\
- & TR & $400-2300 \mathrm{~m}$ \\
- & TR & $0-1500 \mathrm{~m}$ \\
TV & & \\
TV/TD & TR & $0-800 \mathrm{~m}$ \\
\hline
\end{tabular}

$\begin{array}{ccc}\text { TV } & \text { TF } & 2200-2300 \mathrm{~m} \\ \text { F } & \text { TP } & 0-3000 \mathrm{~m} \\ \text { TV/F } & \text { TR } & 0-1100 \mathrm{~m} \\ \text { TV } & \text { TR } & 0-2000 \mathrm{~m} \\ & & \\ \text { TV } & \text { TP } & 0-1000 \mathrm{~m}\end{array}$

$\begin{array}{lll}\text { TV/F } & \text { TR } & 0-2400 \mathrm{~m} \\ \text { TV/F } & \text { TR } & 0-1000 \mathrm{~m}\end{array}$

\author{
Neotrópico e África; \\ AP, AM, PE, BA, RJ, SP, PA e ES \\ Neotropical; RJ e SP \\ Neotropical; \\ RR, AM, PA, CE, PE, BA, GO, \\ MT, MG, ES, RJ, SP, PR, SC e RS \\ Neotropical;
}

AM, PA, PE, AL, BA, MT, MG, ES, RJ, SP, PR, SC e RS

$$
\begin{gathered}
\text { Neotropical; } \\
\text { BA, MG, RJ, SP, SC e RS } \\
\text { Neotropical; BA, ES, SP e RJ } \\
\text { Neotropical; } \\
\text { AM, BA, GO, MG, ES, RJ e SP } \\
\text { Neotropical; BA e RJ } \\
\text { Neotrópico e África; } \\
\text { AM, AP, PA, PE, SE, BA, MG, ES, } \\
\text { RJ, SP, PR, SC e RS }
\end{gathered}
$$

América tropical; BA, ES e RJ

Pantropical;

AM, PA, PE, BA, RJ e SP Neotropical;

AC, PE, BA, MG, RJ, SP e RS Neotropical;

BA, PE, GO, MG, ES, RJ, SP, SC e RS Neotropical;

RR, AC, PA, PE, SE, BA, MG, ES, RJ, SP, SC e RS

$$
\text { Neotropical; }
$$

PE, BA, ES, RJ, SP e RS Neotropical;

RO, AP, AM, PA, BA, PE, RJ, SP, PR e SC 
Tabela 1 (continuação)

\begin{tabular}{|c|c|c|c|c|}
\hline Famílias/Espécies & Substrato & $\begin{array}{l}\text { Forma de } \\
\text { crescimento }\end{array}$ & $\begin{array}{l}\text { Variação altitudinal } \\
\text { no Brasil }\end{array}$ & $\begin{array}{l}\text { Distribuição mundial } \\
\text { e no Brasil }\end{array}$ \\
\hline Cheilolejeunea acutangula (Nees) Grolle & TV & TR & $0-2300 \mathrm{~m}$ & $\begin{array}{c}\text { Neotropical; } \\
\text { AM, PA, BA, PE, MG, ES, RJ e SP }\end{array}$ \\
\hline Cheilolejeunea clausa (Nees \& Mont.) R.M. Schust. & t. TV & TR & $0-800 \mathrm{~m}$ & $\begin{array}{c}\text { Neotropical; } \\
\text { AM, PA, BA, PE, MG, GO, } \\
\text { MT, RJ e SP }\end{array}$ \\
\hline $\begin{array}{l}\text { Cheilolejeunea oncophylla (Ångstr.) Grolle \& } \\
\text { E. Reiner }\end{array}$ & TV & TR & $300-2000 \mathrm{~m}$ & $\begin{array}{c}\text { Neotropical; } \\
\text { RR, BA, MG, RJ, SP e PR }\end{array}$ \\
\hline Cheilolejeunea trifaria (Reinw. et al.) Mizut. & $\mathrm{TV} / \mathrm{F}$ & TR & $0-1000 \mathrm{~m}$ & $\begin{array}{c}\text { Pantropical; } \\
\text { RR, AC, AM, PA, PE, BA, GO, } \\
\text { MT, MG, ES, RJ, SP e PR }\end{array}$ \\
\hline *Cololejeunea obliqua (Nees \& Mont.) Schiffn. & $\mathrm{F}$ & $\mathrm{TP}$ & $0-300 \mathrm{~m}$ & $\begin{array}{c}\text { Neotropical; } \\
\text { AM, PA, PE, BA, RJ, SP, PR e SC }\end{array}$ \\
\hline *Colura tortifolia (Nees \& Mont.) Steph. & $\mathrm{F}$ & $\mathrm{TP}$ & $0-250 \mathrm{~m}$ & Neotropical; AC, PA, BA, SP e RJ \\
\hline $\begin{array}{l}\text { Cyclolejeunea convexistipa (Lehm. \& Lindenb.) } \\
\text { A. Evans }\end{array}$ & $\mathrm{F}$ & $\mathrm{TP}$ & $0-800 \mathrm{~m}$ & Neotropical; AP, AM, PA, BA e SP \\
\hline Cyclolejeunea luteola (Spruce) Grolle & TV & TR & $0-1200 \mathrm{~m}$ & Neotropical; AM, PA, BA, SP e RJ \\
\hline $\begin{array}{l}\text { *Cyclolejeunea peruviana (Lehm. \& Lindenb.) } \\
\text { A. Evans }\end{array}$ & $\mathrm{F}$ & $\mathrm{TP}$ & $0-900 \mathrm{~m}$ & Neotropical; SP \\
\hline *Diplasiolejeunea brunnea Steph. & $\mathrm{F}$ & $\mathrm{TP}$ & $0-800 \mathrm{~m}$ & $\begin{array}{c}\text { Neotropical; } \\
\text { AM, RO, AC, PA, BA, ES, RJ e SC }\end{array}$ \\
\hline Diplasiolejeunea pellucida (Meissn.) Schiffn. & $\mathrm{F}$ & $\mathrm{TP}$ & $0-1000 \mathrm{~m}$ & $\begin{array}{cl}\text { Neotropical; } \\
\text { AM, PA, BA, PE, MG, ES, RJ, } \\
\text { SP, PR e SC }\end{array}$ \\
\hline Drepanolejeunea crucianella (Tayl.) A. Evans & $\mathrm{F}$ & TR & Altitudes baixas & Neotropical; AM, PA e BA \\
\hline Drepanolejeunea fragilis Bischl. & $\mathrm{TV} / \mathrm{F}$ & $\mathrm{TP}$ & 0-1000 m & $\begin{array}{c}\text { Neotropical; } \\
\text { AM, PA, BA, PE, ES e SP }\end{array}$ \\
\hline *Drepanolejeunea lichenicola (Spruce) Steph. & $\mathrm{F}$ & TP & $500-2000 \mathrm{~m}$ & Neotropical; BA, RJ, SP e PR \\
\hline *Drepanolejeunea orthophylla (Nees \& Mont.) Bischl. & hl. F & TR & $0-900 \mathrm{~m}$ & Neotropical; AM, BA, RJ, SP e SC \\
\hline Harpalejeunea subacuta A. Evans & TV & TR & $2000-2400 \mathrm{~m}$ & Neotropical; BA, MG e SP \\
\hline Lejeunea filipes Spruce & TV & TR & $800 \mathrm{~m}$ & Neotropical; BA \\
\hline Lejeunea phyllobola Nees \& Mont. & TV & TR & $0-950 \mathrm{~m}$ & $\begin{array}{c}\text { Neotropical; } \\
\text { AC, AM, PA, BA, ES, RJ, SP e RS }\end{array}$ \\
\hline Lejeunea flava (Sw.) Nees & $\mathrm{TV} / \mathrm{F}$ & TR & $0-2400 \mathrm{~m}$ & $\begin{array}{c}\text { Pantropical; } \\
\text { RR, AC, AM, PA, PE, GO, } \\
\text { BA, MG, ES, RJ, SP e RS }\end{array}$ \\
\hline Leptolejeunea elliptica (Lehm. \& Lindenb.) Schiffn. & $\mathrm{TV} / \mathrm{F}$ & $\mathrm{TP}$ & $0-1500 \mathrm{~m}$ & $\begin{array}{c}\text { Neotropical; } \\
\text { RR, AP, AC, AM, PA, AL, PE, } \\
\text { BA, MG, ES, RJ, SP, PR e SC }\end{array}$ \\
\hline Leucolejeunea conchifolia (A. Evans) A. Evans & TV & TR & $0-1200 \mathrm{~m}$ & $\begin{array}{c}\text { Disjunta (U.S.A. e } \\
\text { Neotrópico); BA, ES e SP }\end{array}$ \\
\hline Leucolejeunea unciloba (Lindenb.) A. Evans & TV & TR & $0-1300 \mathrm{~m}$ & $\begin{array}{c}\text { Neotropical; } \\
\text { PE, BA, ES, RJ, SP, SC e RS }\end{array}$ \\
\hline Leucolejeunea xanthocarpa (Lehm. \& Lindenb.)A. Evans & s TV & TR & $0-2500 \mathrm{~m}$ & $\begin{array}{c}\text { Pantropical; } \\
\text { PE, BA, MG, ES, RJ, SP e SC }\end{array}$ \\
\hline Lopholejeunea subfusca (Nees) Schiffn. & TV & TR & $0-750 \mathrm{~m}$ & $\begin{array}{c}\text { Pantropical; } \\
\text { RR, RO, AC, AM, PA, PB, } \\
\text { PE, BA, ES, RJ e SP }\end{array}$ \\
\hline Marchesinia brachiata (Sw.) Schiffn. & TD & TR & $0-1500 \mathrm{~m}$ & $\begin{array}{c}\text { Neotropical; } \\
\text { RR, PE, BA, SE, MT, MG, } \\
\text { ES, RJ, SP, PR e SC }\end{array}$ \\
\hline Metalejeunea cucullata (Reinw. et al.) Grolle & TV & TR & $0-1350 \mathrm{~m}$ & Pantropical; BA, RJ e SP \\
\hline Microlejeunea bullata (Tayl.) Steph. & TV & TR & $0-2400 \mathrm{~m}$ & $\begin{array}{c}\text { Neotropical; } \\
\text { AC, RR, BA, SE, ES, RJ e SP }\end{array}$ \\
\hline Microlejeunea epiphylla Bischl. & $\mathrm{F}$ & TR & $0-2050 \mathrm{~m}$ & $\begin{array}{l}\text { Disjunta: U.S.A. e Neotrópico; } \\
\text { PE, PB, BA, MG e SP }\end{array}$ \\
\hline
\end{tabular}


Tabela 1 (continuação)

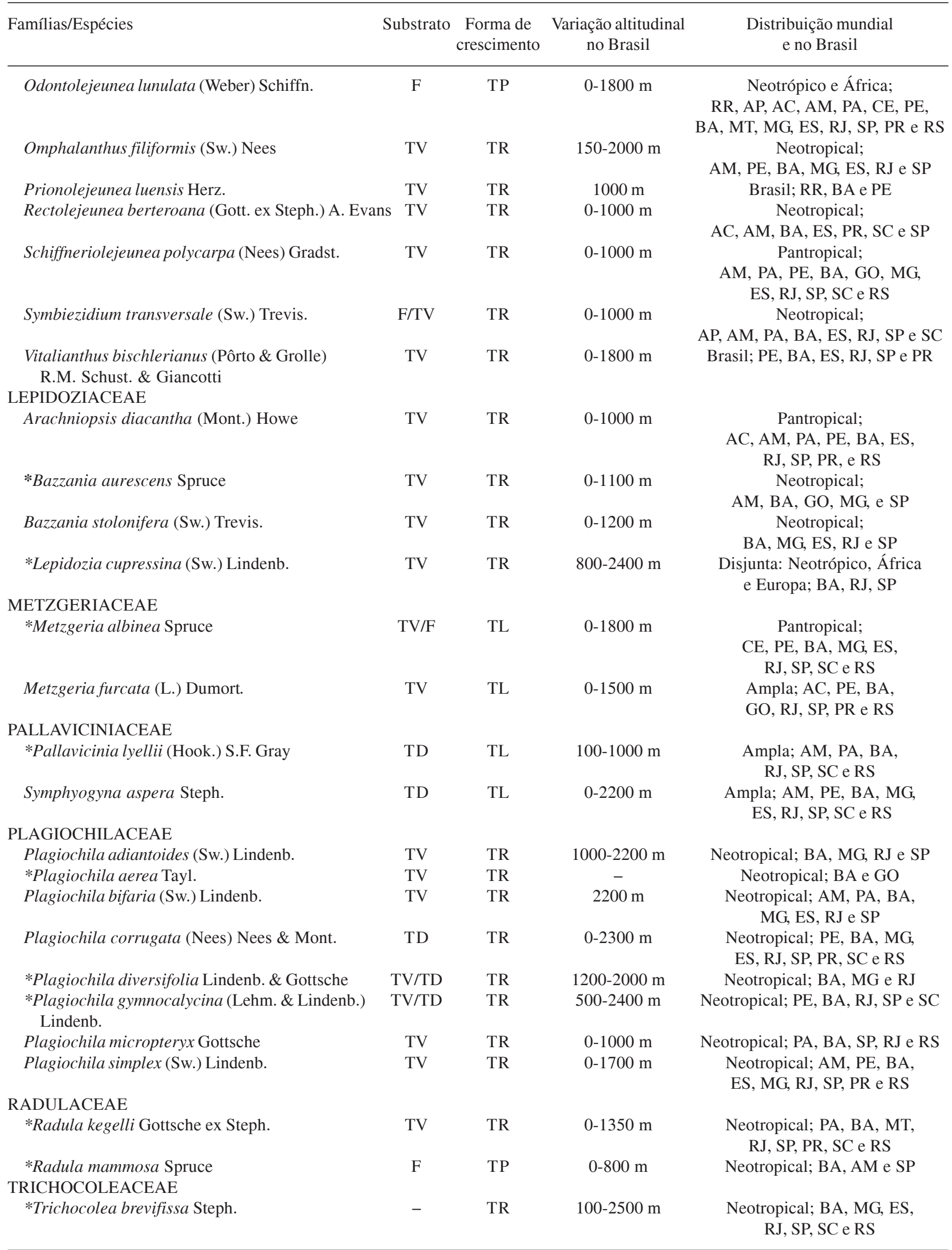


montana, apresentando ca. $45 \%$ da diversidade total de espécies. Em seguida vem Plagiochilaceae, com $11 \%$ das espécies, sendo três novas ocorrências para o Estado, e Jubulaceae, com 6\%.

Os resultados florísticos obtidos apresentaram uma considerável relação com aqueles de remanescentes de Mata Atlântica da região Sudeste do país e com a flora da região Amazônica.

As hepáticas na área de estudo colonizaram três tipos de substrato: tronco vivo, folhas e tronco em decomposição. Não foram encontradas espécies sobre o solo. A escassez de briófitas colonizando este substrato em florestas tropicais com altitude abaixo de 1.500 m é comentada por Richards (1988) e Gradstein (1995). Os autores afirmam que o fato pode ser atribuído a grande quantidade de folhas mortas caídas, a temperatura elevada e a baixa luminosidade que dificultam um balanço fotossintético positivo e conseqüentemente o desenvolvimento das briófitas neste substrato. A ocorrência de briófitas terrícolas nesse bioma deve-se, em grande parte, à presença de espécies em barrancos úmidos à margem da floresta ou ao longo de trilhas ou mesmo em clareiras no seu interior (Richards 1988; Pôrto 1992; Gradstein 1995). O substrato rocha é raro no fragmento florestal estudado e, quando presente, apresenta-se colonizado apenas por musgos.

Corticícola foi a comunidade mais expressiva, sendo representada por 47 espécies (67\%), dentre as quais 34 são exclusivas à colonização sobre tronco vivo. Seguem-se as epífilas, com 23 espécies (33\%), sendo 14 exclusivas e, finalmente, as epíxilas com 10 espécies (14\%), das quais seis são exclusivas (Fig. 1). Os dados obtidos assemelham-se aos relatados para florestas tropicais úmidas, em relação à colonização preferencial de hepáticas tanto sobre tronco como sobre folha (Richards 1984, 1988; Gradstein \& Pócs 1989). É

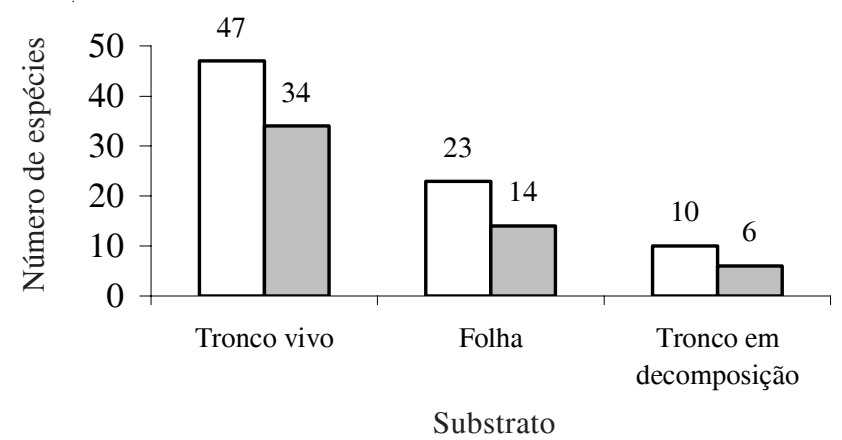

Figura 1. Substratos colonizados pelas espécies de hepáticas da Serra da Jibóia, Município de Santa Teresinha, Bahia, Brasil. $\square=$ Substrato; $\square=$ Exclusividade por substrato. importante destacar a considerável representatividade da comunidade epífila, conhecida por suas características peculiares, exigência de alta umidade e vulnerabilidade a qualquer impacto destrutivo na estrutura da floresta (Richards 1984; Pócs 1996; Gradstein 1997).

A colonização de briófitas sobre folhas é uma característica de florestas tropicais úmidas (Richards 1984). Briófitas tipicamente epífilas são, em sua maioria, hepáticas da família Lejeuneaceae (subfamília, Lejeuneoideae), além de algumas espécies de Radula (seção Epiphyllae). As epífilas são um grupo especial que possui adaptações à colonização das folhas como o tamanho reduzido, coloração verde claro, aderência ao substrato através de discos adesivos originados da fusão dos rizóides e reprodução assexual através de gemas (Richards 1984; Gradstein 1997). Estas características podem ser observadas nos táxons citados por Gradstein (1997) que também ocorreram na área de estudo: Cololejeunea spp., Cyclolejeunea spp., Colura spp., Diplasiolejeunea spp., Drepanolejeunea spp., Leptolejeunea spp., Microlejeunea spp., Odontolejeunea spp. e Radula spp.

Cyclolejeunea peruviana e $C$. convexistipa estão muito bem representadas neste substrato, colonizando grande número de amostras, e em alguns casos cobrindo toda a lâmina foliar.

Cinco tipos de forma de crescimento foram caracterizados: pendente, tapete, talosa, trama, e tufo. Houve predominância da forma trama com 48 espécies $(68,6 \%)$, enquanto que 13 espécies $(18,6 \%)$ são caracterizadas como tapete, seis como talosa $(8,6 \%)$, duas como pendente $(2,8 \%)$ e uma como tufo $(1,4 \%)$.

Uma única forma de crescimento foi observada cada espécie. Este resultado era esperado já que nas florestas tropicais úmidas a diversidade de formas de crescimento em musgos, em geral, é maior do que em hepáticas (Richards 1984). Trama foi predominante nas espécies da Serra da Jibóia, sendo bem representada nas famílias Lejeuneaceae, Lepidoziaceae e Plagiochilaceae. Esta forma de crescimento é típica deste tipo de ambiente e coloniza preferencialmente substratos como troncos vivos e folhas, porque promove maior fixação, facilitando o maior contato com o substrato e, conseqüentemente, ampliando a superfície de absorção (Mägdefrau 1982; Pôrto 1992). Resultados semelhantes foram relatados por Pôrto (1992), Costa (1999) e Molinaro \& Costa (2001), entre outros.

Em relação à variação altitudinal, os táxons inventariados na Serra da Jibóia correspondem aos 
característicos de florestas tropicais baixo montana, de acordo com Gradstein et al. (2001), a saber os gêneros Bazzania, Calypogeia, Cephalozia, Diplasiolejeunea, Herbertus, Lepidozia, Leptoscyphus, Lophocolea, Metzgeria, Odontoschisma, Plagiochila, Symphyogyna, Trichocolea e a espécie Riccardia fucoidea. Ainda concordando com os referidos autores, também estiveram representados táxons característicos de floresta tropical submontana a exemplo de: Colura, Vitalianthus, Frullania, Marchesinia, Calypogeia, Pallavicinia, Arachniopsis. Também houve uma menor ocorrência de táxons característicos de florestas secas e savanas: Frullania caulisequa, Schiffneriolejeunea polycarpa e Leucolejeunea spp.

A variação altitudinal foi ampliada para as espécies Cololejeunea obliqua, Colura tortifolia e Drepanolejeunea crucianela, anteriormente referidas para altitudes de 0-300 m (Gradstein \& Costa 2003).

Das 70 espécies citadas para a Serra da Jibóia, 22 $(31,4 \%)$ foram classificadas como raras. Dentre elas, Lejeunea filipes, até o momento, era conhecida apenas para o estado da Bahia, além de Cyclolejeunea peruviana, Leptoscyphus gibbosus e Plagiochila aerea, que foram citadas para um estado brasileiro, respectivamente, São Paulo, Rio de Janeiro e Goiás. Merecem destaque ainda, Lepidozia cupressina, Plagiochila diversifolia, Radula mammosa e Riccardia fucoidea, que possuíam referências apenas para dois estados cada, e Cephalozia crassifolia, Drepanolejeunea crucianela, Drepanolejeunea lichenicola, Harpalejeunea subacuta, Herbertus juniperoideus, Leucolejeunea conchifolia, Metalejeunea cucullata e Prionolejeunea luensis, que eram referidas para apenas três estados brasileiros (Tab. 1).

Trinta e seis $(51,4 \%)$ espécies apresentaram uma distribuição moderada e apenas $12(17,1 \%)$ espécies têm distribuição ampla no Brasil. Também foi assinalada para a área, Vitalianthus bischlerianus, espécie endêmica do Brasil (Gradstein \& Costa 2003).

A flora de hepáticas estudada apresentou os seguintes padrões de distribuição: Neotropical $(71,4 \%$, ou 50 spp.), Pantropical (12,9\% ou 9 spp.), Cosmopolita, Neotrópico e África, Disjunto (U.S.A. e Neotrópico; África, Neotrópico e Europa - 4,3\% ou 3 spp.) e Restrito ao Brasil (2,9\% ou 2 spp.).

São citadas para o Estado da Bahia por Gradstein \& Costa (2003) 120 espécies. Os resultados de trabalhos mais recentes (Bastos \& Yano 2002; 2003; C.J.P. Bastos, dados não publicados) e o presente levantamento totalizam 210 espécies para o Estado, ou seja, $43 \%$ de aumento em relação ao conhecimento anterior. Isso vem reforçar a necessidade de continuidade dos estudos sobre a brioflora baiana, especialmente em remanescentes de Mata Atlântica onde a diversidade é alta.

No caso particular da Serra da Jibóia é urgente a realização do levantamento dos musgos que, a exemplo das hepáticas, certamente se farão representar por uma considerável riqueza taxonômica.

Em síntese, a rica flora de hepáticas que veio a ser conhecida nessa área, sobretudo da comunidade epífila, assim como o elevado número de táxons de distribuição rara a moderada, e a ocorrência de uma espécie endêmica do Brasil, são indicadores de uma condição de integridade ambiental satisfatória e baixa ação antrópica na Serra da Jibóia e respaldam a necessidade de se empreender esforços no sentido de transformá-la em uma Unidade de Conservação, de modo que a biodiversidade existente seja efetivamente preservada.

\section{Referências bibliográficas}

Bastos C.J.P.; Albertos, B. \& Vilas Bôas, S.B. 1998a. Bryophytes from some caatinga areas in the State of Bahia (Brazil). Tropical Bryology 14: 69-75.

Bastos C.J.P.; Stradmann, M.T. \& Vilas Bôas-Bastos, S.B. 1998b. Additional contribution to the bryophyte flora from Chapada Diamantina National Park, State of Bahia, Brazil. Tropical Bryology 15: 15-20.

Bastos, C.J.P. \& Vilas Bôas-Bastos, S.B. 2000a. Ocurrence of some Lejeuneaceae (Jungermaniophyta) in Bahia Brazil. Tropical Bryology 18: 45-54.

Bastos, C.J.P. \& Vilas Bôas-Bastos, S.B. 2000b. Some new additions to the hepatic flora (Jungermaniophyta) for the state of Bahia Brazil. Tropical Bryology 18: 1-11.

Bastos, C.J.P. \& Vilas Bôas-Bastos, S.B. 2000c. Variações morfológicas do lóbulo de Lejeunea glaucescens Gott. (Lejeuneaceae, Hepticopsida). Acta Botanica Malacitana 25: 73-80.

Bastos, C.J.P. \& Yano, O. 2002. Pycnolejeunea porrectilobula (Lejeuneaceae), a new species from Brazil. Nova Hedwigia 74(3-4): 439-443.

Bastos, C.J.P. \& Yano, O. 2003. New records of the genus Rectolejeunea (Lejeuneaceae) for the state of Bahia, Brazil. Nova Hedwigia 76(3-4): 477-485

Bastos, C.J.P.; Yano, O. \& Vilas Bôas-Bastos, S.B. 2000. Briófitas de campos rupestres da Chapada Diamantina, Estado da Bahia, Brasil. Revista Brasileira de Botânica 23(4): 357-368.

Bernecker-Lücking, A. 1999. Key to Latin American species of Bazzania S.F. Gray. Tropical Bryology 16: 117-126.

Bischler, H. 1962. The genus Calypogeja Raddi in South America II. Subgenus Calypogeja, subgroups 1, 2 and 3. Candollea 18: 53-93. 
Bischler, H. 1964. Le genre Drepanolejeunea Steph. em Amérique Centrale et Méridionale. Revue Bryologique et Liquénologique 33(1-2): 15-179.

Bischler, H. 1967. Le genre Drepanolejeunea Steph. em Amérique Centrale et Méridionale. Revue Bryologique et Liquénologique 35(1-4): 95-134.

Bravo, F. 2001a. Sete novas espécies de Trichomyia (Diptera, Psychodidae) da Mata Atlântica do Nordeste do Brasil. Sitientibus série Ciências Biológicas 1(2): 121-130.

Bravo, F. 2001b. Brunettia bora, a new species of Moth-fly (Diptera, Psychodidae) from Brazil. Studies on Neotropical Fauna and Environment 36(3): 211-214.

Bravo, F. 2002. Novas espécies de Trichomyia (Diptera, Psychodidae) da Mata Atlântica da Bahia, Nordeste do Brasil. Iheringia, Série Zoologia 92(3): 57-67.

Bravo, F. 2003. Cinco espécies novas de Caenobrunettia (Diptera: Psychodidae, Psychodinae) do Brasil. Neotropical Entomology 32(2): 279-285.

Bravo, F. 2004. Espécie nova de Paracetomima Duckhouse (Diptera Psichodidae) do Nordeste Brasileiro. Revista Brasileira de Zoologia 21(2): 281-282.

Costa, D.P. 1999. Epiphytic bryophyte diversity in primary and secondary lowland rainforests in Southeastern Brazil. The Bryologist 102(2): 320-326.

Costa, D.P. \& Moura A.C. 1996. Metzgeriaceae (Hepaticopsida) de Nova Friburgo, Estado do Rio de Janeiro, Brasil. Hoehnea 23(1): 103-122.

Crandall-Stotler B. \& Stotler R.E. 2000. Morphology and classification of the Marchantiophyta. Pp. 21-70. In: A.J. Shaw \& B. Goffinet (eds.). Bryophyte Biology. Cambridge, Cambridge University Press.

Fulford, M. 1963. Manual of Hepaticae of Latin America. Part I. Memories of The New York Botanical Garden 11(1): 1-172.

Fulford, M. 1966. Manual of Hepaticae of Latin America. Part II. Memories of The New York Botanical Garden 11(2): 173-276.

Fulford, M. 1968. Manual of Hepaticae of Latin America. Part III. Memories of The New York Botanical Garden 11(3): 277-392.

Góes-Neto, A.; Marques, M.F.O.; Andrade, J.D. \& Santos, D.S. 2003. Lignicolous aphyllophoroid Basidiomycota in an Atlantic Forest Fragment in the semi-arid caatinga region of Brazil. Mycotaxon 88: 359-364.

Gouvêa, J.B.S.; Mattos-Silva, L.A. \& Hori, M. 1976. Fitogeografia. In: Diagnóstico sócio-econômico da região cacaueira. Comissão executiva do plano da Lavoura Cacaueira 7: 1-7.

Gradstein, S.R. 1989. A key to the Hepaticae and Anthocerotae of Puerto Rico and Virgin Islands. Bryologist 92(3): 329-348.

Gradstein, S.R. 1995. Bryophyte diversity of the tropical rainforest. Archs Science Genève 48(1): 91-96.

Gradstein, S.R. 1997. The taxonomic diversity of epiphyllous bryophytes. Abstracta Botanica 21(1): 15-19.

Gradstein, S.R.; Churchill, S.P. \& Salazar Allen, N. 2001. Guide to the bryophytes of Tropical America. Memoirs of The New York Botanical Garden 86: 1-577.
Gradstein, S.R. \& Costa, D.P. 2003. Liverworts and hornworts of Brazil. Memoirs of The New York Botanical Garden 87: 1-301.

Gradstein, S.R. \& Pócs, T. 1989. Bryophytes. Pp. 311-325. In: H. Lieth \& M.J.A. Werger (eds.). Tropical Rain Forest Ecosystems. Amsterdam, Elsevier Science Publischers B.V.

Grolle, R. \& Reiner-Drehwald, M.E. 1997. Cheilolejeunea oncophylla (Angstr.) Grolle \& Reiner comb. nov. (Lejeuneaceae), from the Neotropics. Journal of Bryology 19: 781-75.

Harley, R.M. 1995. Bryophyta. Pp. 803-812. In: B.L. Stannard (ed.). Flora of the Pico das Almas. Chapada Diamantina - Bahia, Brazil. Kew, Royal Botanic Garden.

Heinrichs J.; Gradstein, S.R. \& Grolle, R. 1998. A revision of the neotropical species of Plagiochila (Dumort.) Dumort. (Hepaticae) described by Olof Swartz. Journal of the Hattori Botanical Laboratory 85: 1-32.

Heinrichs J.; Anton, H.; Gradstein, S.R. \& Mues, R. 2000. Systematics of Plagiochila sect. Glaucescentes Carl (Hepaticae) from tropical America: a morphological and chemotaxonomical approach. Plant Systematic and Evolution 220: 115-138.

Hell, K.G. 1969. Briófitas talosas dos arredores da cidade de São Paulo (Brasil). Universidade de São Paulo. Boletim da Faculdade de Filosofia, Ciências e Letras 25: 1-190.

Ilkiu-Borges, A.L. \& Lisboa, R.C.L. 2002a. Os gêneros Cyrtolejeunea Evans e Drepanolejeunea Steph. (Lejeuneaceae) na Estação Científica Ferreira Penna (PA) e novas ocorrências. Boletim do Museu Paraense Emílio Goeldi, série Botânica 18(2): 231-245.

Ilkiu-Borges, A.L. \& Lisboa, R.C.L. 2002b. Os gêneros Lejeunea e Microlejeunea (Lejeuneaceae) na Estação Científica Ferreira Penna, Estado do Pará, Brasil, e novas ocorrências. Acta Amazonica 32(4): 541-553.

Lemos-Michel, E.L. 2001. Hepáticas epifíticas sobre o Pinheiro-Brasileiro no Rio Grande do Sul. Porto Alegre, Ed. Universidade/UFRGS.

Lemos-Michel, E.L. 1983. Frullania (Jungermanniales, Hepaticopsida) no Rio Grande do Sul, Brasil. I. Subgênero Diastaloba. Revista Brasileira de Botânica 6(2):115-123.

Lemos-Michel, E.L. \& Yano, O. 1998. O gênero Bryopteris (Hepatophyta) no Brasil. Acta Botanica Brasilica 12(1): $5-24$.

Mägdefrau, K. 1982. Life-forms of bryophytes. Pp. 45-58 In: Bryophyte Ecology. A.J.E. Smith (ed.). Chapman and Hall. Cambridge, Cambridge University Press.

Molinaro, L.C. \& Costa, P.C. 2001. Briófitas do arboreto do Jardim Botânico do Rio de Janeiro. Rodriguésia 52(81): 107-124.

Oliveira-e-Silva, M.I.M.N. \& Yano, O. 2000. Anthocerothophyta e Hepatophyta de Mangaratiba e Angra dos Reis, Rio de Janeiro, Brasil. Boletim do Instituto de Botânica 13: 1-102.

Onraedt, M. 1985. Bryophytes de Sri Lanka VII. Lejeuneaceae Holostipae. Cryptogamie, Bryologique Lichénologique 9(1): 51-62. 
Onraedt, M. 1988. Contribution a la Flore Bryologique de Guyane Française. III. Cryptogamie, Bryologique Lichénologique 6(2): 151-175.

Pócs, T. 1996. Epiphyllous liwerwort diversity at worldwide level and its threat and conservation. Anales de Instituto de Biología de Universidad Nacional Autónoma de México. Serie Botanica 67(1): 109-127.

Pôrto, K.C. 1992. Bryoflores d'une forêt de plaine et d'une forêt d'altitude moyenne dans l'état de Pernambuco (Brésil). 2. Analyse écologique comparative des forêts. Cryptogamie, Bryologique Lichénologique 13(3): 187-219.

Queiroz, L.P.; Sena, T.S.N. \& Costa, M.J.S.L. 1996. Flora vascular da Serra da Jibóia, Santa Terezinha-Bahia. I: O Campo Rupestre. Sitientibus 15: 27-40.

Reiner-Drehwald, M.E. 1994. El género Radula Dum. (Radulaceae, Hepaticae) en el Noreste da Argentina. Tropical Bryology 9: 5-22.

Reiner-Drehwald, M.E. 1998. Las Lejeuneaceae (Hepaticae) de Misiones, Argentina V. Cheilolejeunea y Lepidolejeunea. Tropical Bryology 14: 53-68.

Reiner-Drehwald, M.E. 2000. Las Lejeuneaceae (Hepaticae) de Misiones, Argentina VI. Lejeunea y Taxilejeunea. Tropical Bryology 19: 81-131.
Reyes, D.M. 1982. El Género Diplasiolejeunea en Cuba. Acta Botanica Academiae Scientiarum Hungaricae 28(1-2): 145-180.

Richards, W.P. 1984. The ecology of tropical forest bryophytes. Pp. 1233-1270. In: R.M. Schuster (ed.). v.2. New Manual of Bryology.

Richards, W.P. 1988. Tropical forest bryophytes. Synusiae and strategies. The Journal Hattori Botanical Laboratory 64: 1-4.

Teeuwen, M. 1989. A revision of genus Odontolejeunea (Spruce) Schiffn. (Lejeuneaceae, Hepaticae). Nova Hedwigia 48(1-2): 1-32.

Veloso, H.P. \& Góes Filho, L. 1982. Fitogeografia brasileira: classificação fisionômica-ecológica da vegetação neotropical. Boletim técnico (Série vegetação) № 1,80 p. Salvador, IBGE.

Vilas Bôas-Bastos, S.B. \& Bastos, C.J.P. 1998. Briófitas de uma área de cerrado no município de Alagoinhas, Bahia, Brasil. Tropical Bryology 15: 101-110.

Yano, O. 1989. Briófitas. Pp. 27-30. In: O. Fidalgo \& V.L.R. Bononi (eds.). Técnicas de coleta, preservação e herborização de material botânico. Série Documentos. São Paulo, Instituto de Botânica. 